05,04

\title{
Магнитные и магнитоэлектрические свойства скандобората $\mathrm{NdSc}_{3}\left(\mathrm{BO}_{3}\right)_{4}$
}

\author{
(C) E.В. Еремин ${ }^{1,2,3}$, А.А. Дубровский ${ }^{1}$, И.А. Гудим ${ }^{1}$, В.Р. Титова ${ }^{1,2}$, М.В. Меркулов ${ }^{2}$ \\ ${ }^{1}$ Институт физики им. Л.В. Киренского, ФИЦ КНЦ СО РАН, \\ Красноярск, Россия \\ ${ }^{2}$ Сибирский фредеральный университет, \\ Красноярск, Россия \\ ${ }^{3}$ Сибирский государственный университет науки и технологий им. М.Ф. Решетнева, \\ Красноярск, Россия \\ E-mail: eev@iph.krasn.ru
}

Поступила в Редакцию 3 марта 2021 г.

В окончательной редакции 3 марта 2021 г.

Принята к публикации 8 марта 2021 г.

Групповым методом из раствора-расплава на основе тримолибдата висмута выращены монокристаллы тригонального неодимового скандобората $\mathrm{NdSc}_{3}\left(\mathrm{BO}_{3}\right)_{4}$. Исследованы его магнитные и магнитоэлектрические свойства в диапазоне температур $4.2-300 \mathrm{~K}$ и в магнитных полях до $9 \mathrm{~T}$. В магнитном отношении он является парамагнетиком, свойства которого определяются магнитными свойствами иона $\mathrm{Nd}^{3+} \mathrm{c}$ учетом ванфлековской парамагнитной поправки. Величина магнитоэлектрической поляризации оказалась сравнимой с ранее исследованным разбавленным алюмоборатом неодима $\mathrm{Nd}_{0.35} \mathrm{Y}_{0.65} \mathrm{Al}_{3}\left(\mathrm{BO}_{3}\right)_{4}$. Последнее обстоятельство открывает еще один подкласс соединений, в которых должен наблюдаться гигантский магнитоэлектрический эффект.

Ключевые слова: магнитоэлектрический эффект, рост кристаллов, мультферроики.

DOI: 10.21883/FTT.2021.07.51041.042

\section{1. Введение}

Тригональные

редкоземельные

оксибораты $R M_{3}\left(\mathrm{BO}_{3}\right)_{4}(R-$ редкоземельный ион, $M=\mathrm{Al}, \mathrm{Sc}$, $\mathrm{Fe}, \mathrm{Ga})$ в последнее десятилетие активно исследуются благодаря широкой возможности различных комбинаций $R$ - и $M$-элементов и, соответственно, большому разнообразию физических свойств [1-8]. Оксибораты имеют ромбоэдрическую структуру типа природного минарела хантита, описываемую пространственной группой $R 32$ или $P 3_{1} 21$. Нецентросимметричная структура делает эти материалы перспективными кандидатами для оптических приложений, благодаря их хорошим люминесцентным и нелинейным оптическим свойствам. Для ферроборатов $R \mathrm{Fe}_{3}\left(\mathrm{BO}_{3}\right)_{4}$ установлена их принадлежность к новому классу мультиферроиков, в которых сосуществуют магнитные, электрические и упругие параметры порядка.

Интерес к парамагнитным алюмоборатам усилился с обнаружением в $\mathrm{TmAl}_{3}\left(\mathrm{BO}_{3}\right)_{4}$ большого магнитоэлектрического эффекта [9], который оказался сравним по величине с наблюдаемыми эффектами в изоструктурных ферроборатах $R \mathrm{Fe}_{3}\left(\mathrm{BO}_{3}\right)_{4}$. Позже был обнаружен еще больший магнитоэлектрический эффект в $\mathrm{HoAl}_{3}\left(\mathrm{BO}_{3}\right)_{4}[10]$, величина которого $\left(\sim 5480 \mu \mathrm{C} / \mathrm{m}^{2}\right)$ в разы превышает известные максимальные значения магнитоэлектрической поляризации в ферроборатах (в $\mathrm{SmFe}_{3}\left(\mathrm{BO}_{3}\right)_{4} \sim 500 \mu \mathrm{C} / \mathrm{m}^{2}$ [5] и $\mathrm{NdFe}_{3}\left(\mathrm{BO}_{3}\right)_{4}$ около $300 \mu \mathrm{C} / \mathrm{m}^{2}$ [3] и $\mathrm{Ho}_{0.5} \mathrm{Nd}_{0.5} \mathrm{Fe}_{3}\left(\mathrm{BO}_{3}\right)_{4} \sim 900 \mu \mathrm{C} / \mathrm{m}^{2}$ [3]).
Увеличение магнитоэлектрического эффекта наблюдается и при замене в $\mathrm{HoFe}_{3}\left(\mathrm{BO}_{3}\right)_{4}$ ионов $\mathrm{Fe}^{3+}$ на ионы $\mathrm{Ga}^{3+}[11]$.

Принимая во внимание, что при замене в $R M_{3}\left(\mathrm{BO}_{3}\right)_{4}$ магнитной подсистемы $(\mathrm{Fe})$ на немагнитную (Al, Ga) магнитоэлектрический эффект достигает гигантских значений, возникает интерес исследовать и другие подклассы оксиборатов со структурой хантита с одной магнитной подсистемой, например, редкоземельные скандобораты $R \mathrm{Sc}_{3}\left(\mathrm{BO}_{3}\right)_{4}$. В качестве объекта для исследования был выбран скандоборат $\mathrm{NdSc}_{3}\left(\mathrm{BO}_{3}\right)_{4}$. Выбор этого соединения важен еще и тем, что стабильной тригональной фазы $\mathrm{NdAl}_{3}\left(\mathrm{BO}_{3}\right)_{4}$ не существует, и ранее был исследован только замещенный алюмоборат $\mathrm{Nd}_{0.35} \mathrm{Y}_{0.65} \mathrm{Al}_{3}\left(\mathrm{BO}_{3}\right)_{4}$ [12].

В настоящей работе представлены результаты магнитных и магнитоэлектрических исследований скандобората $\mathrm{NdSc}_{3}\left(\mathrm{BO}_{3}\right)$.

\section{2. Подготовка образцов и методика измерений}

Монокристаллы $\mathrm{NdSc}_{3}\left(\mathrm{BO}_{3}\right)_{4}$ выращивались из раствора-расплава массой на основе молибдата висмута по технологии, подробно описанной в работе [13]. Раствор-расплавную систему удобно представить в квазибинарной форме $89 \mathrm{wt} . \%\left[\mathrm{Bi}_{2} \mathrm{Mo}_{3} \mathrm{O}_{12}+3 \mathrm{Li}_{2} \mathrm{O}_{3}+2 \mathrm{~B}_{2} \mathrm{O}_{3}\right.$ $\left.+0.1 \mathrm{Nd}_{2} \mathrm{O}_{3}\right]+11$ wt. $\% \mathrm{NdSc}_{3}\left(\mathrm{BO}_{3}\right)_{4}$. 
Температура насыщения уточнялась $\pm 2^{\circ} \mathrm{C}$ с помощью пробных кристаллов, и для данного раствора-расплава она оказалась равной $980^{\circ} \mathrm{C}$. Кристаллы выращивались на затравках в режиме с понижением температуры $1^{\circ} \mathrm{C} /$ day. Стартовая температура была $T=T_{s}-7^{\circ} \mathrm{C}$. Выращенные кристаллы имели размер порядка 4-6 mm. По завершению процесса выращивания кристаллы охлаждались до комнатной температуры со скоростью не более $100^{\circ} \mathrm{C} / \mathrm{h}$.

Магнитные свойства были исследованы на вибрационном магнитометре PPMS QuantumDesign (центр коллективного пользования ФИЦ КНЦ СО РАН) в диапазоне температур 4.2-300 K и магнитных полях до 9 Т, а также на а также на вибрационном магнетометре оригинальной конструкции [14]. Для измерения магнитоэлектрической поляризации на грани исследуемого образца, приготовленного в виде плоскопараллельной пластинки (плоскости граней перпендикулярны оси второго порядка, $a$-оси) наносились электроды из эпоксидной смолы с проводящим наполнителем. Заряд, возникающий на образце вследствие магнитоэлектрического эффекта, измерялся электрометром Keithley 6517В. Магнитное поле прикладывалось вдоль кристаллографической оси второго порядка ( $a$-оси) и вдоль $b$-оси, перпендикулярной $a$-оси и оси третьего порядка (c-оси).

\section{3. Результаты и обсуждение}

Как было показано ранее при высокой температуре $\mathrm{NdSc}_{3}\left(\mathrm{BO}_{3}\right)_{4}$ имеет ромбоэдрическую структуру, описываемую пространственной группой $R 32$. А при понижении температуры $\left(T_{s}=504 \mathrm{~K}\right)$ испытывает структурный переход $R 32 \rightarrow P 3{ }_{1} 21$ [12].

Исследования магнитных свойств проводились в магнитных полях $B=0.1,1,3,5,6$ и 9Т, при ориентации магнитного поля вдоль $c$-оси третьего порядка и вдоль $a$-оси второго порядка, перпендикулярной $c$-оси. На рис. 1 представлены температурные зависимости намагниченности в направлении магнитного поля вдоль и перпендикулярно оси третьего порядка. Видно, что при намагничивании вдоль $c$-оси при низких температурах имеет место тенденция к насыщению, и как следствие к отклонению от закона Кюри (рис. 2).

Из рис. 1 видно, что для всех кривых зависимости $M(T)$, имеют похожий вид типичный для парамагнетика и различаются лишь по величине. С увеличением поля зависимости $M_{\|}(T)$ и $M_{\perp}(T)$ возрастают с разной скоростью, демонстрируя заметную анизотропию (рис. 3), которая увеличивается с ростом магнитного поля.

В направлениях В $\| c$ намагниченность $M_{\|}(T)$ много меньше $M_{\perp}(T)$, почти линейно зависит от магнитного поля (рис. 4) и имеет форму несвойственную для классических парамагнетиков (рис. $1, a)$. Такой вид $M_{\|}(T)$ может быть следствием примешивания возбужденных состояний иона $\mathrm{Nd}^{3+}$ к основному. Т. е наряду с ориен-
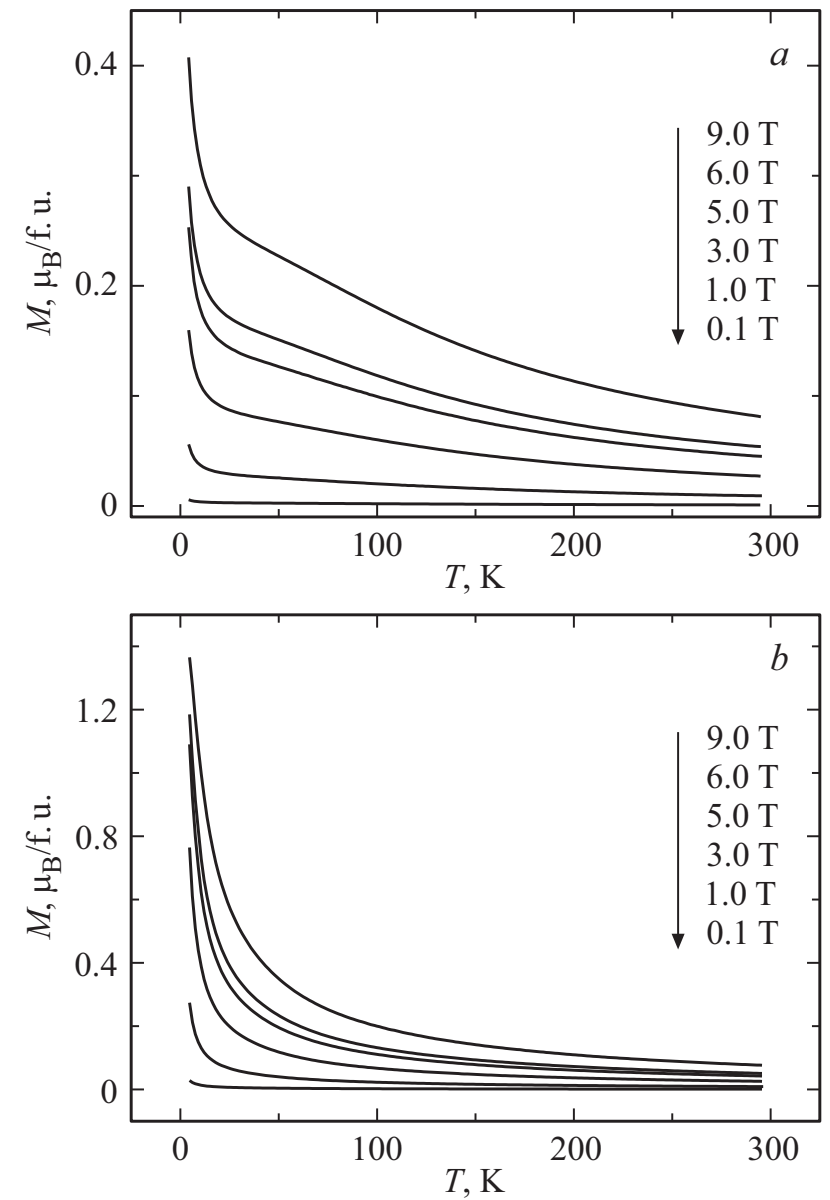

Рис. 1. Температурные зависимости намагниченности $\mathrm{NdSc}_{3}\left(\mathrm{BO}_{3}\right)_{4}$, измеренные в различных магнитных полях в геометрии $\mathbf{B} \| c(a)$ и $\mathbf{B} \perp c(b)$.

тационным парамагнетизмом необходимо еще учитывать поправку Ван Флека в определении магнитного момента.

Используя закон Кюри-Вейса, из высокотемпературной зависимости магнитной восприимчивости можно определить парамагнитную температуру Кюри $\theta$ и эффективный магнитный момент иона $\mathrm{Nd}^{3+}$ в элементарной ячейке. На рис. 2 показана такая зависимость полученная в магнитном поле $\mathbf{B}=0.1 \mathrm{~T}$ в различной геометрии эксперимента $(\mathbf{B} \| c$ и $\mathbf{B} \perp c)$. Эффективны магнитный момент одной структурной единицы $\mathrm{NdSc}_{3}\left(\mathrm{BO}_{3}\right)_{4}$ оказался равен $\mu_{\mathrm{eff}}=3.69 \mu_{\mathrm{B}}$, что близко к теоретическому значению $\mu_{\text {eff }}$ с учетом поправки парамагнетизма Ван Флека $\left(\mu_{\mathrm{eff}}=3.68 \mu_{\mathrm{B}}\right)$. Тогда как теоретическое значение магнитного момента иона $\mathrm{Nd}^{3+}$, определяемого как $\mu=g \mu_{\mathrm{B}} \sqrt{J(J+1)}$ (где $g=8 / 11-$ $g$-фактор иона $\mathrm{Nd}^{3+}$ в свободном состоянии) равно $\mu_{\mathrm{Nd}}=3.62 \mu_{\mathrm{B}}$.

На рис. 5 изображены полевые зависимости поперечной $\left(\Delta P_{a b}\left(B_{b}\right)\right)$ и продольной $\left(\Delta P_{a a}\left(B_{a}\right)\right)$ магнитоэлектрической поляризации $\mathrm{NdSc}_{3}\left(\mathrm{BO}_{3}\right)_{4}$ при различных температурах. Видно, что с ростом поля наблюдается нелинейный рост кривых $\Delta P_{a b}$ и $\Delta P_{a a}$. Такой ход кривых 


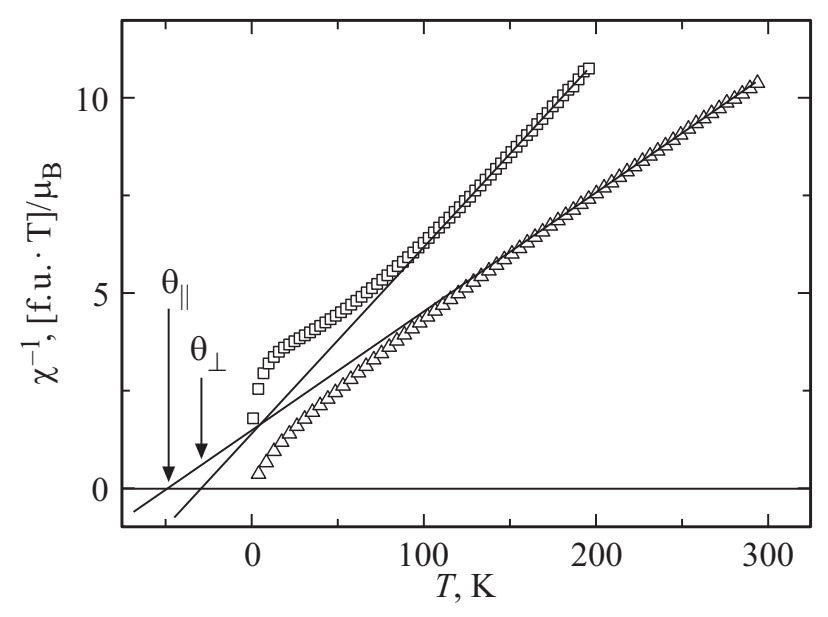

Pис. 2. Обратная зависимость магнитной восприимчивости $\mathrm{NdSc}_{3}\left(\mathrm{BO}_{3}\right)_{4}$ от температуры, измеренная в магнитном поле 9 Т в геометрии $\mathbf{B} \| c$ (треугольники) и $\mathbf{B} \perp c$ (квадраты).

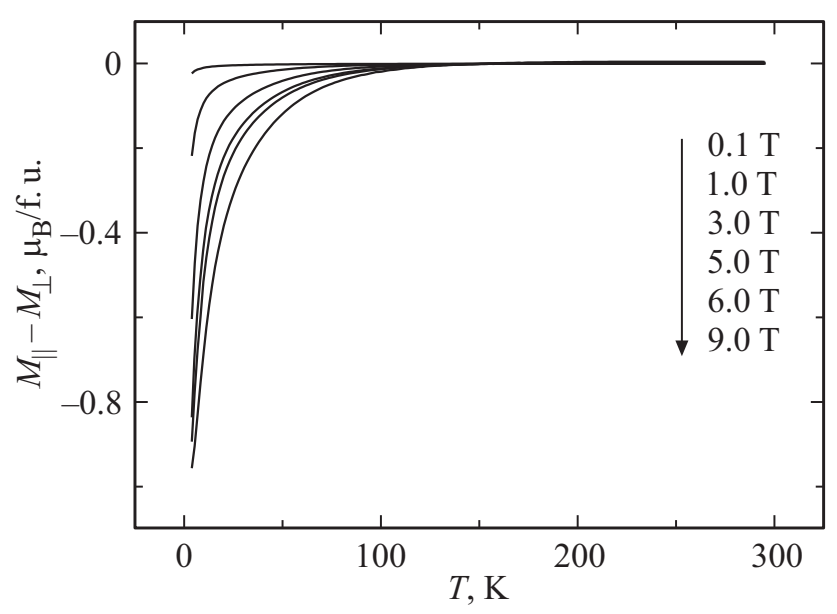

Рис. 3. Температурные зависимости $M_{\|}-M_{\perp} \mathrm{NdSc}_{3}\left(\mathrm{BO}_{3}\right)_{4}$, полученные в различных магнитных полях.

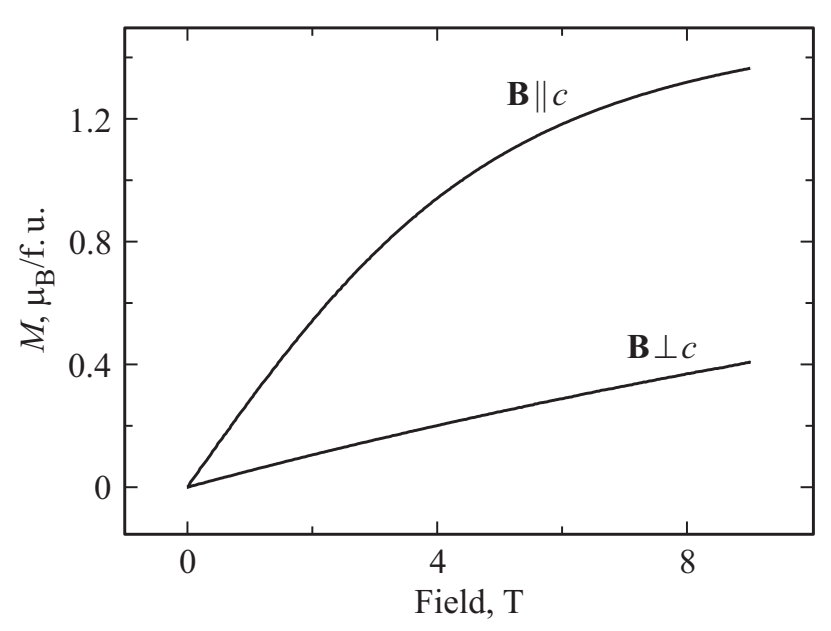

Рис. 4. Полевые зависимости намагниченности $\mathrm{NdSc}_{3}\left(\mathrm{BO}_{3}\right)_{4}$, полученные при $T=4.2 \mathrm{~K}$ в геометрии $\mathbf{B} \| c$ и $\mathbf{B} \perp c$.

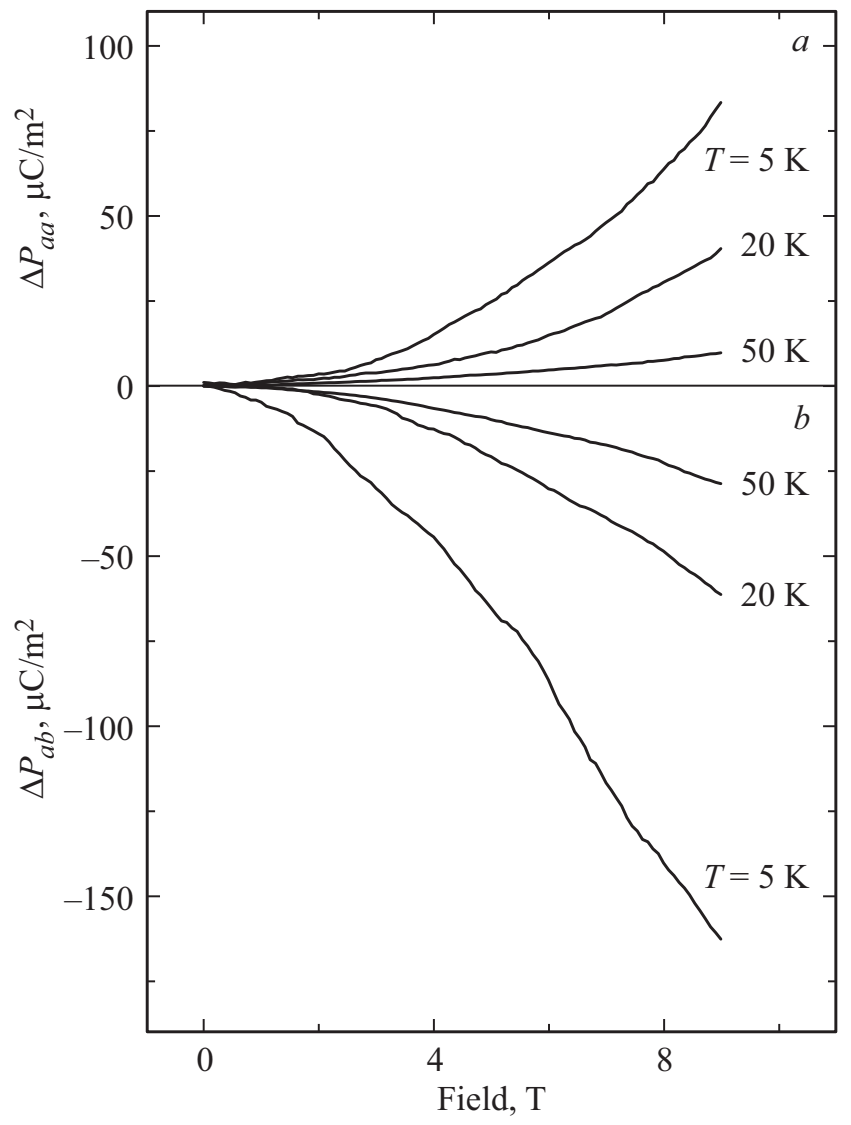

Рис. 5. Полевые зависимости продольной магнитоэлектрической поляризации $\Delta P_{a}$ при различных температурах: $a-$ в геометрии $\mathbf{B} \| a, b-$ в геометрии $\mathbf{B} \| b$.

магнитоэлектрической поляризации является типичным для парамагнитных хантитов [9-11].

Обнаружена существенная анизотропия магнитоэлектрической поляризации от направления магнитного поля. Из рис. 5 видно, что величина поперечной поляризации достигает значений $\Delta P_{a b}\left(B_{b}\right) \sim-163 \mu \mathrm{C} / \mathrm{m}^{2}$, а продольной $\Delta P_{a a}\left(B_{a}\right) \sim 83 \mu \mathrm{C} / \mathrm{m}^{2}$ при температуpe $T=5 \mathrm{~K}$ и полях $9 \mathrm{~T}$. Эти значения больше по сравнению с ранее исследованным алюмоборатом неодима $\mathrm{Nd}_{0.35} \mathrm{Y}_{0.65} \mathrm{Al}_{3}\left(\mathrm{BO}_{3}\right)_{4}\left(\Delta P_{a b}\left(B_{b}\right) \sim-68 \mu \mathrm{C} / \mathrm{m}^{2}\right.$, $\Delta P_{a a}\left(B_{a}\right) \sim 69 \mu \mathrm{C} / \mathrm{m}^{2}$ при $T=5 \mathrm{~K}$ и $\left.H=9 \mathrm{~T}\right)$. Однако, если учесть, что концентрация иона $\mathrm{Nd}^{3+}$ в алюмоборате в $1 / 0.35 \approx 3$ раза меньше, чем в скандоборате неодима, то можно предположить, что увеличение эффекта при замене $\mathrm{Al}\left(\mathrm{B} \mathrm{Nd}_{0.35} \mathrm{Y}_{0.65} \mathrm{Al}_{3}\left(\mathrm{BO}_{3}\right)_{4}\right)$ на $\mathrm{Sc}\left(\right.$ в $\left.\mathrm{NdSc}_{3}\left(\mathrm{BO}_{3}\right)_{4}\right)$ объясняется за счет увеличения концентрации магнитоактивного иона $\mathrm{Nd}^{3+}$. С другой стороны, ранее было показано, что магнитоэлектрический эффект может быть выше ожидаемого за счет изменения энергетической структуры редкоземельного иона, вызванного локальными искажениями [15]. Появление локальных искажений в $\mathrm{Nd}_{0.35} \mathrm{Y}_{0.65} \mathrm{Al}_{3}\left(\mathrm{BO}_{3}\right)_{4}$ очевидно имеет место из-за разного ионного радиуса $\mathrm{Al}^{3+}$ и $\mathrm{Y}^{3+}$ и неравномерного распределения ионов в матрице кристалла. Таким обра- 
зом, в настоящее время нельзя сказать какая матрица $R \mathrm{Al}_{3}\left(\mathrm{BO}_{3}\right)_{4}$ или $R \mathrm{Sc}_{3}\left(\mathrm{BO}_{3}\right)_{4}$ даст наибольший эффект. Для этого необходимы дополнительные исследования. Однозначно можно утверждать лишь то, что ионы $\mathrm{Sc}^{3+}$ наряду с ионами $\mathrm{Al}^{3+}$ и $\mathrm{Ga}^{3+}$ дают гигантский магнитоэлектрический эффект в редкоземельных оксиборатах со структурой хантита $R M_{3}\left(\mathrm{BO}_{3}\right)_{4}(R-$ редкоземельный ион, $M=\mathrm{Al}, \mathrm{Sc}, \mathrm{Ga})$.

\section{4. Заключение}

Групповым методом из раствора-расплава на основе тримолибдата висмута был выращен скандоборат $\mathrm{NdSc}_{3}\left(\mathrm{BO}_{3}\right)_{4}$. Впервые исследованы его магнитные и магнитоэлектрические свойства в диапазоне температур $4.2-300 \mathrm{~K}$ и магнитных полях до $9 \mathrm{~T}$.

В магнитном отношении $\mathrm{NdSc}_{3}\left(\mathrm{BO}_{3}\right)_{4}$ является парамагнетиком, свойства которого целиком определяются магнитным поведением тона $\mathrm{Nd}^{3+}$. Определены парамагнитная температура Кюри $\left(\theta_{\perp}=-28 \mathrm{~K}, \theta_{\|}=48 \mathrm{~K}\right)$ и эффективный момент $\mu_{\text {eff }}=3.69 \mu_{\mathrm{B}}$ на формульную единицу. Показано, что $\mu_{\mathrm{eff}}$ близко к теоретическому значению $\mu_{\text {eff }}$ с учетом вклада парамагнетизма Ван Флека $\left(\mu_{\text {eff }}=3.68 \mu_{\mathrm{B}}\right)$.

Обнаружена существенная анизотропия магнитоэлектрической поляризации от направления магнитного поля $\Delta P_{a b}\left(B_{b}\right) \sim-163 \mu \mathrm{C} / \mathrm{m}^{2}$, а $\Delta P_{a a}\left(B_{a}\right) \sim 83 \mu \mathrm{C} / \mathrm{m}^{2}$ при температуре $T=5 \mathrm{~K}$ в полях $9 \mathrm{~T}$. В сравнении с ранее исследованным разбавленным алюмоборатом неодима $\mathrm{Nd}_{0.35} \mathrm{Y}_{0.65} \mathrm{Al}_{3}\left(\mathrm{BO}_{3}\right)_{4}$ эффект в $\mathrm{NdSc}_{3}\left(\mathrm{BO}_{3}\right)_{4}$ меньше, однако это может быть вызвано изменением энергетической структуры редкоземельного иона $\mathrm{Nd}^{3+}$ в $\mathrm{Nd}_{0.35} \mathrm{Y}_{0.65} \mathrm{Al}_{3}\left(\mathrm{BO}_{3}\right)_{4}$ вызванного локальными искажениями из-за разного ионного радиуса $\mathrm{Al}^{3+}$ и $\mathrm{Y}^{3+}$ и неравномерного распределения ионов в матрице кристалла.

\section{Финансирование работы}

Исследование выполнено при финансовой поддержке Российского фонда фундаментальных исследований, Правительства Красноярского края, Красноярского краевого фонда науки в рамках научного проекта: Разработка раствор-расплавных технологий выращивания новых монокристаллов скандоборатов со структурой хантита и исследование взаимодействия их магнитной и электрической подсистем (№ 18-42-240011).

\section{Конфликт интересов}

Авторы заявляют, что у них нет конфликта интересов.

\section{Список литературы}

[1] А.К. Звездин, С.С. Кротов, А.М. Кадомцева, Г.П. Воробьев, Ю.Ф. Попов, А.П. Пятаков, Л.Н. Безматерных, Е.А. Попова. Письма в ЖЭТФ 81, 6, 335 (2005).
[2] E.A. Popova, D.V. Volkov, A.N. Vasiliev, A.A. Demidov, N.P. Kolmakova, I.A. Gudim, L.N. Bezmaternykh, N. Tristan, Yu. Skourski, B. Buechner, C. Hess, R. Klingeler. Phys. Rev. B 75, 22, 224413 (2007).

[3] R.P. Chaudhury, F. Yen, B. Lorenz, Y.Y. Sun, L.N. Bezmaternykh, V.L. Temerov, C.W. Chu. Phys. Rev. B 80, 10, 104424 (2009).

[4] А.М. Кадомцева, Ю.Ф. Попов, Г.П. Воробьев, А.П. Пятаков, С.С. Кротов, К.И. Камилов, В.Ю. Иванов, А.А. Мухин, А.К. Звездин, А.М. Кузьменко, Л.Н. Безматерных, И.А. Гудим, В.Л. Темеров. ФНТ 36, 6, 640 (2010).

[5] В.И. Зиненко, М.С. Павловский, А.С. Крылов, И.А. Гудим, Е.В. Еремин. ЖЭТФ 144, 6, 1174 (2013).

[6] А.П. Пятаков, А.К. Звездин. УФН 182, 6, 593(2012).

[7] А.Н. Васильев, Е.А. Попова. ФНТ 32, 8-9, 968 (2006).

[8] T. Usui, Y. Tanaka, H. Nakajima, M. Taguchi, A. Chainani, M. Oura, S. Shin, N. Katayama, H. Sawa, Y. Wakabayashi, T. Kimura. Nature Mater. 13, 6, 618.

[9] R.P. Chaudhury, B. Lorenz, Y.Y. Sun, L.N. Bezmaternykh, V.L. Temerov, C.W. Chu. Phys. Rev. B 81, 22, 220402 (2010).

[10] K.-C. Liang, R.P. Chaudhury, B. Lorenz, Y.Y. Sun, L.N. Bezmaternykh, V.L. Temerov, C.W. Chu. Phys. Rev. B 83, 18, 180417 (2011).

[11] Н.В. Волков, И.А. Гудим, Е.В. Еремин, И.А. Бегунов, А.А. Демидов, К.Н. Болдырев. Письма в ЖЭТФ 99, 2, 72 (2014).

[12] E.V. Eremin, M.S. Pavlovskiy, I.A. Gudim, V.L. Temerov, M.S. Molokeev, N.D. Andryushin, E.V. Bogdanov. J. Alloys Compd. 828, 154355 (2020).

[13] L.N. Bezmaternykh, V.L. Temerov, I.A. Gudim, N.A. Stolbovaya. Crystallogr. Rep. 50, 1, S97 (2005).

[14] А.Д. Балаев, Ю.В. Бояршинов, М.И. Карпенко, Б.П. Хрусталёв. ПТЭ 3, 167 (1985)

[15] E. Eremin, I. Gudim, V. Temerov, D. Smolyakov, M. Molokeev. J. Cryst. Growth 518, 1 (2020).

Редактор Д.В. Жуманов 